\title{
Graphene Related Nanostructures Synthesized by High-Energy Ball Milling
}

\author{
I. Estrada-Guel ${ }^{1,2}$, O. Anderson-Okonkwo ${ }^{1}$, F.C. Robles-Hernandez ${ }^{1}$ \\ 1. Department of Mechanical Engineering Technology, University of Houston, Houston, TX 77204- \\ 4020, USA \\ 2. Centro de Investigación en Materiales Avanzados (CIMAV). Laboratorio Nacional de Nanotecnología \\ Miguel de Cervantes No. 120, 31109, Chihuahua, Chih., México
}

The size and structure of the nanoparticulate materials imparts unique characteristics [1] perceptible at macro scale, leading ours to a new universe of possible areas of application. A good example is carbon allotropes as fullerenes, graphenes, etc. These structures have been the focus of research looking for new synthesis routes and potential applications. One important problem with its synthesis is the generation of soot in form of amorphous carbon. Soot is considered a byproduct that in some cases requires tedious, expensive and wasteful procedures for removing. On the other hand, soot can be visualized as the precursor of fullerenes or technically defined as the black solid product of incomplete combustion or pyrolysis of fossil fuels and other organic materials. This material plays important roles as industrial filler and pigment (carbon black) and a traffic-related air pollutant (diesel soot) [2]. It is primarily composed of carbon (>80\%) in form of agglomerated primary particles with diameters on the order of 10-30 nm and crystalline and amorphous domains. In spite of the wide interest in fullerenes, very little is known about structures found in soots, the relation between them or their stability. It is known that some structural changes of soot particles may also be induced by laser irradiation [3]. On the other hand, carbon nanostructures (e.g. graphenes, nanotubes, etc.) are synthesized by evaporation methods. Here in this research we present alternative methods to synthesize this nanostructures using solid state methods such as high-energy ball milling that are cost effective and environmentally safe. For carbon, the crystallinity changes with ball milling time the general conclusion is that milling induces nanocrystalline-amorphous phase transitions [4]. However, there exist few reports upon the effect of milling over amorphous and carbon graphitic structures [5].

In this work, some evidence of microstructural changes of soots after high-energy ball milling is presented. The raw material is commercially available fullerene soot in form of a byproduct from fullerene synthesis. Soots were milled using a SPEX 8000M for intervals from 0 to 10h. After milling, samples were characterized by Raman spectrometry in a Horiba-XploRA device and morphological studies were performed via SEM in a JSM-7201F and TEM with a JEM2200-FS microscope.

The Fig. 1 shows two SEM-TEM micrographs of original soot (0h) and as-milled samples. Raw material (Fig. 1a) presents wooly particles characteristic of evaporation residues. These particles form agglomerates and clusters. The TEM observations show that the soot particles observed by SEM are agglomerates with limited_crystallinity. The particles observed in the soot are in the range of 20-50 $\mathrm{nm}$. After milling (Fig. 1b), the morphology of the particles changes abruptly in form of laminar aggregates with domains lower than $20 \mathrm{~nm}$.

Raman studies show that as the sample is milled, the D and G band begins to develop indicating the fact that mechanical milling is capable of inducing crystallization of carbon soot into complex structures such as graphitic carbon and graphene. These in-situ changes are induced by the high energetic energy released by the impacts of milling media. The high intensity in the $2 \mathrm{D}$ band in milled samples indicates that graphitic carbon was fractured leading to the development of graphene layers. The TEM micrograph 
shown in Fig. 3 corroborates the presence of stacked graphene sheets, showing an interplanar spacing of $0.335 \mathrm{~nm}$.

\section{References:}

[1] B. Munkhbayar et al. P. Tech 234 (2013) p. 132-140.

[2] A. Sadezky et al. Carbon 43 (2005) p. 1731-1742.

[3] L. Hu et al. Carbon 44 (2006) p. 1725-1729.

[4] C.P. Marshall, M.A. Wilson. Carbon 42 (2004) p. 2179-2186.

[5] A.D. Lueking et al. Carbon 45 (2007) p. 2297-2306.

[6] I. Estrada-Guel thanks the CONACYT support under project 169262 and the Redes Temáticas de Nanociencias y Nanotecnología (124886).
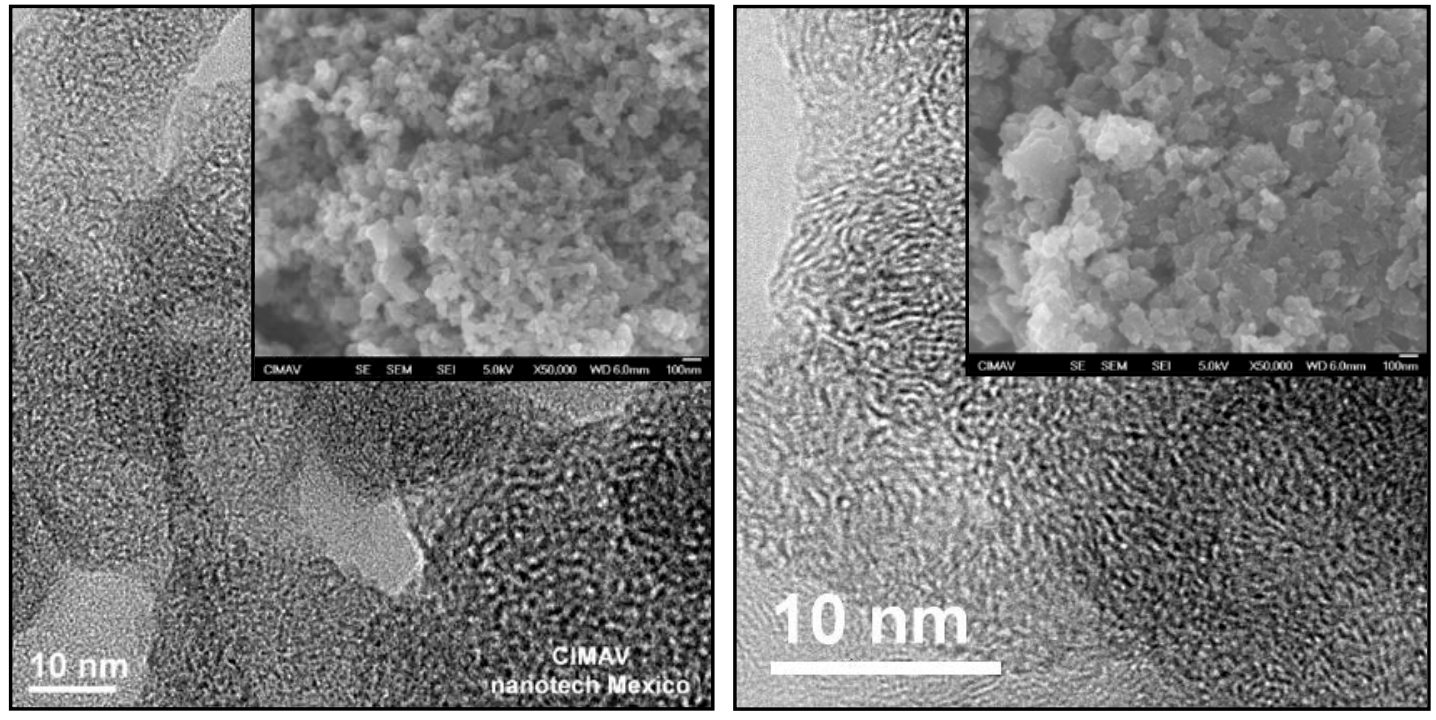

Figure 1. SEM-TEM micrographs of a) Raw soot (0h) and b) sample after 4h of milling.
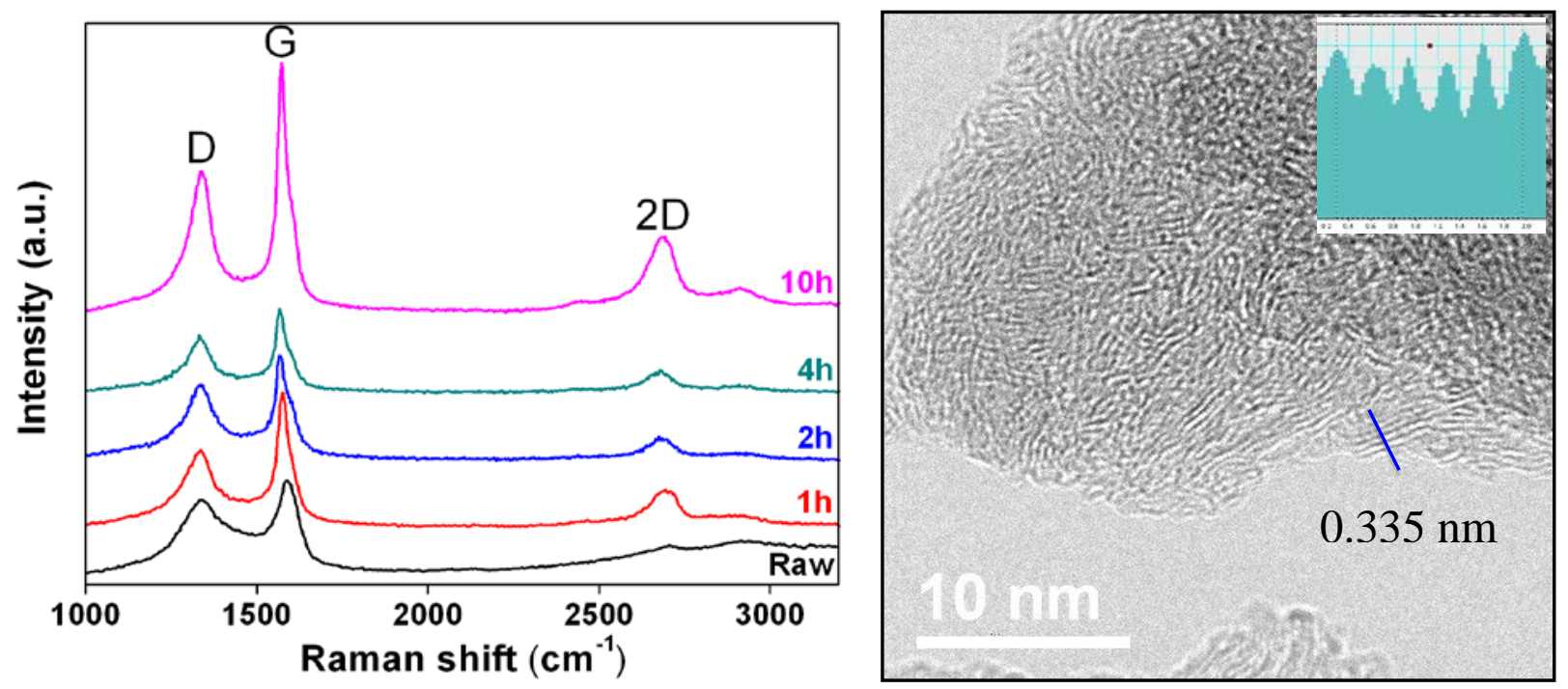

Figure 2. Raman spectra of soot samples as a function of milling time and HRTEM image of $4 \mathrm{~h}$ milled sample. 